\title{
Editorial
}

\section{Advanced Technologies for Mobile IoT and Cyber-Physical Systems}

\author{
Kyungtae Kang, ${ }^{1}$ Kyung-Joon Park, ${ }^{2}$ Qixin Wang, ${ }^{3}$ and Wenyao Xu ${ }^{4}$ \\ ${ }^{1}$ Department of Computer Science \& Engineering, Hanyang University, Ansan 15588, Republic of Korea \\ ${ }^{2}$ Department of Information and Communication Engineering, DGIST, Daegu 42988, Republic of Korea \\ ${ }^{3}$ Department of Computing, The Hong Kong Polytechnic University, Hong Kong \\ ${ }^{4}$ Department of Computer Science \& Engineering, State University of New York, Buffalo, NY 14260, USA
}

Correspondence should be addressed to Kyungtae Kang; ktkang@hanyang.ac.kr

Received 18 August 2016; Accepted 18 August 2016

Copyright (C) 2016 Kyungtae Kang et al. This is an open access article distributed under the Creative Commons Attribution License, which permits unrestricted use, distribution, and reproduction in any medium, provided the original work is properly cited.

Recently, cyber-physical systems (CPS) have emerged as a promising research paradigm. This development is the convergence of control, communication, and computation. As computing and communication capabilities have become both faster and cheaper, we can expect to find them embedded in diverse objects and structures in the physical environment. This provides the basis for applications that link the "cyberworld" of computing and communications with the physical world, thereby promising great potential for significant social and economic impact. Unlike traditional embedded systems that primarily focus on computing elements, CPS also takes into account the physical, real-world components that interact with computing elements in cyber space. Examples of CPS encompass a broad range of complex man-made systems such as avionics, vehicles, transportation, healthcare, smart grid systems, and other fields.

In the US, the President's Council of Advisors on Science and Technology has recommended CPS as a top priority for federal research investments. As a result, the CPS program was initiated at the National Science Foundation (NSF) with a funding level of around 30 million USD per year in 2009. In addition, during the past few years, the NSF has sponsored Workshops on CPS and related fields. Most recently, in 2010, the ACM and the IEEE have jointly launched the First International Conference on Cyber-Physical Systems (ICCPS), which was very successful with an impressive number of submitted papers.

However, so far, most of these initial research efforts on CPS have been made by the real-time and embedded systems community. For example, ICCPS was cosponsored by the ACM Special Interest Group on Embedded Systems (SIGBED) and the IEEE Technical Committee on RealTime Systems (TCRTS). As mentioned above, since the salient feature of CPS is tight integration between cyber and physical components, it is obvious that networking (or mobile computing) plays a key role in the coordination between the cyber and physical elements of CPS. In fact, CPS applications are necessarily promoted by the "Internet of Things" (IoT) architectures and protocols. This combination of IoT and CPS facilitates the collection, management, and processing of large datasets, as well as the support of complex processes to manage and control physical systems at different scales.

This special issue aims to identify emerging research topics in CPS, especially from the mobile communication and networking perspective, and to define the future of networking research in CPS. In particular, by properly addressing complex interactions between the cyber and physical elements of CPS, we focus on challenging issues in IoT research that need to be resolved for deriving eventual working solutions for CPS. This facilitates timely dissemination of stateof-the-art research on CPS to the networking and mobile computing research communities.

Mobile IoT/CPS is concerned with the provision of IoT-enabled CPS for mobile objects and devices. Mobile internet devices, such as the iPhone and Android phones, with their increasing processing power, range of sensors, and pervasive cellular connections, already provide ubiquitous platforms for building robust, reliable, and secure mobile IoT/CPS applications. Therefore, the objective of this special issue is also to contribute to the direction of research on 


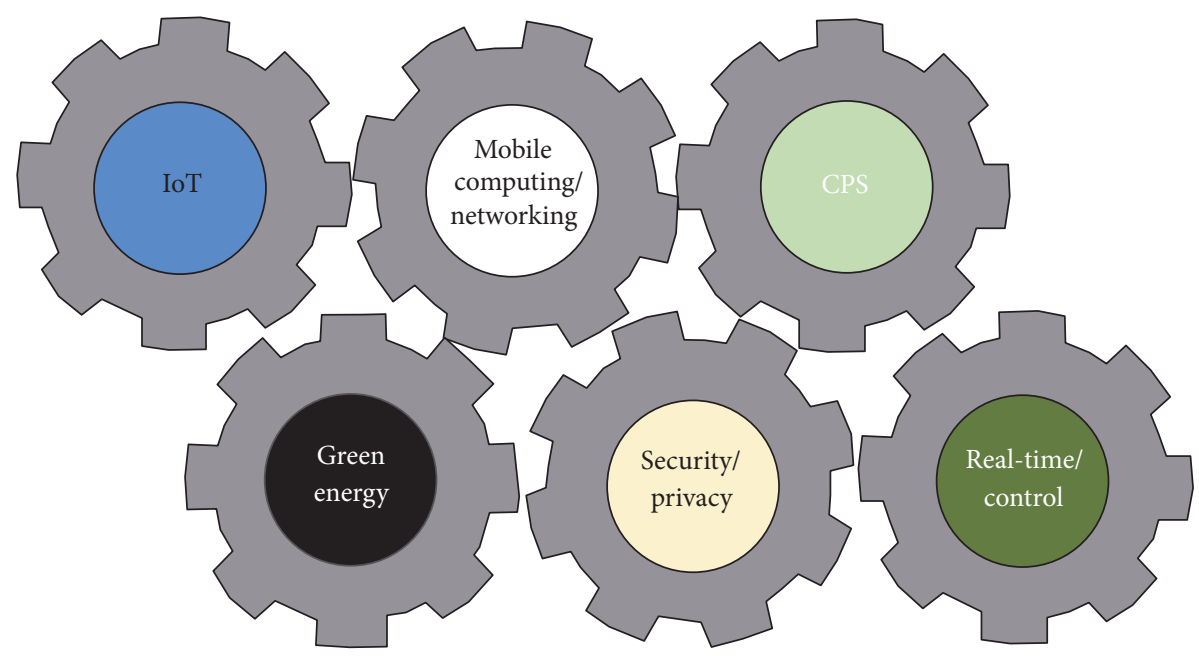

FIGURE 1: Major areas of research focus in this special issue.

mobile IoT/CPS by addressing issues critical to mobility, from advances in the underlying science to the challenges of development and implementation. The primary areas of research on which this special issue focuses are depicted in Figure 1.

We received 25 papers, of which 6 papers were accepted after a rigorous review process. Among these, the first paper entitled "A Dynamic Programming Solution for EnergyOptimal Video Playback on Mobile Devices" by M. Song and J. Park proposes an algorithm that determines the CPU frequency needed to decode each frame in a video. The aim is to minimize power consumption while meeting buffer size and deadline constraints using a dynamic programming technique. The proposed scheme finds the sequence of frequencies by taking into account the frame decoding time, decoding deadlines, and the measured active and idle power consumption of a system. Experimental results based on measurements made on a physical device show that it saves an appreciable amount of energy, which provides useful guidelines for a low-power video service by providing the minimum bound on the power consumption required for video playback.

Next, the paper entitled "A Remote Medical Monitoring System for Heart Failure Prognosis" by L. Zhang et al. presents the design and implementation of a remote medical monitoring system for heart failure (HF) prediction. The system realized early prediction (or prognosis) of future HF occurrence by estimating future NT-proBNP level based on a patient's historical data (body weight and blood pressure) where the data were obtained remotely using noninvasive devices (i.e., a Bluetooth-based weight scale and sphygmomanometer). A Heart Failure Risk Score (HFRS) was proposed to evaluate the risk of future occurrence of $\mathrm{HF}$ based on the prediction results. The HFRS scoring was designed so that it could be easily understood and perceived by patients. This system optimizes early-stage delivery of multiple suggestions/interventions to patients at different risk levels. This end-to-end system can also be used to manage patients and their data by doctors or by the system's data center. To validate this system, a set of real-life data from 34 patients was collected in a pilot clinical trial. The proposed $\mathrm{HF}$ prediction algorithms achieved an overall accuracy of $79.4 \%$ and $67.6 \%$ when using data collected over a 30 -day and 7-day period, respectively. Therefore, from a clinical perspective, this system is promising in reducing the morbidity and mortality caused by HF and therefore improves clinical outcomes.

The third paper entitled "Dynamic Vehicular Route Guidance Using Traffic Prediction Information” by K. Kim et al. proposes a dynamic vehicular routing algorithm with traffic prediction for improved routing performance. The primary idea of the proposed algorithm is to use both real-time and predictive traffic information provided by a central routing controller. In order to evaluate the performance, the authors develop a microtraffic simulator that provides road networks created from real maps, routing algorithms, and vehicles that travel from origins to destinations depending on traffic conditions. The performance is evaluated by a newly defined metric that reveals the travel time distributions more accurately than the commonly used metric of mean travel time. Simulation results show that the proposed dynamic routing algorithm with prediction outperforms both the static and dynamic types without prediction routing algorithms under various traffic conditions and road configurations. Traffic scenarios are included where not all vehicles comply with the proposed dynamic routing with a prediction strategy. Even under these conditions, the results suggest that more than half the benefit of the new routing algorithm is realized even when only $30 \%$ of vehicles comply.

The fourth paper entitled "Energy-Efficient Real-Time Human Activity Recognition on Smart Mobile Devices" by J. Lee and J. Kim presents a novel approach for the human activity recognition (HAR) process that dynamically controls the activity recognition duration for energy-efficient HAR. Conventional HARs using the built-in accelerator in smart mobile devices are known to be the most energy-efficient in 
that field, but they still incur high power consumption due to the sensor operation itself as well as the accompanying CPU computation overhead. To further enhance the energy efficiency, the proposed approach first classifies a user's activities as static and dynamic and controls the classification duration and sleep time for the HAR process accordingly based on two factors, the acceleration-sampling frequency and the window size. The experimental results showed that the proposed approach reduced energy consumption by a minimum of about $44.23 \%$ and a maximum of about $78.85 \%$ compared to conventional HAR (such as the support vector machine) without sacrificing accuracy. Moreover, this paper reports on how the acceleration-sampling frequency, window size, and feature vector dimensionality alter the battery power consumption behavior with HAR.

The fifth paper entitled "Control-Scheduling Codesign Exploiting Trade-Off between Task Periods and Deadlines" by H.-J. Cha et al. proposes a novel task set synthesis algorithm that exploits the trade-off relation between a control task's period and the deadline for maximizing the overall system control performance. This paper first shows how the control task period and the deadline affect the control performance; that is, the shorter period and shorter deadline both enhance the control task's control performance. From this observation, the authors conduct a measurement study using a Lane Keeping Assist System control application, which gives the control performance profile of the task for each possible period/deadline pair. Next, assuming multiple similar tasks with their control profiles, the paper defines a period and deadline selection problem that optimizes the overall control performance, that is, the sum of the control performance of the control tasks in the system. For this optimization problem, the authors propose a heuristic algorithm that finds a high-quality suboptimal solution with very low complexity, which makes the proposed solution practically applicable to large task sets.

The last paper entitled "Efficient Attribute-Based Secure Data Sharing with Hidden Policies and Traceability in Mobile Health Networks" by C. Hahn et al. presents a novel technique to efficiently and securely share data in mobile healthcare systems. The proposed solution enables the data owner to encrypt data and to attach an attribute-based policy of interest to the cipher text. The proposed solution hides a description of the policy so that not only the data but also the policy is available only to authorized users. The proposed solution enables the size of any cipher text to remain constant, irrespective of the number of attributes. The proposed solution embeds a user-specific identifier into each decryption key so that the identity of a user can be traced in case of malicious activities, for example, illegal key sharing.

\section{Acknowledgments}

We sincerely thank the editorial board for their approval of this concept and continuous help in successful publication of this special issue. We would also like to thank the contributors to this special issue for their innovative work. We extend our thanks to the reviewers for critical assessment of each paper, their constructive criticism, and timely responses that made this special issue possible. This work was supported by the research fund of Hanyang University (HY-2014-P).

Kyungtae Kang Kyung-Joon Park Qixin Wang Wenyao Xu 

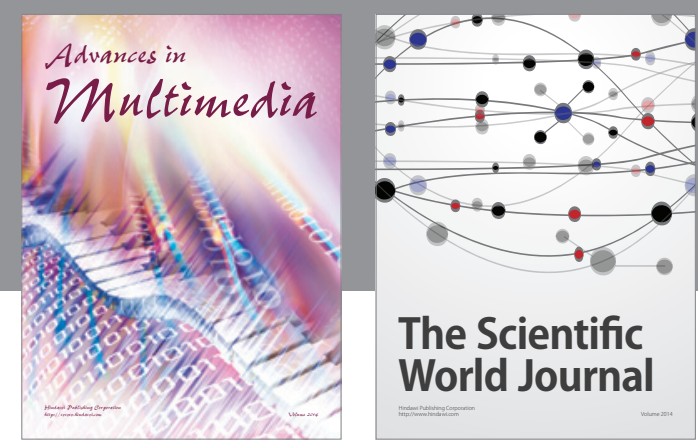

The Scientific World Journal
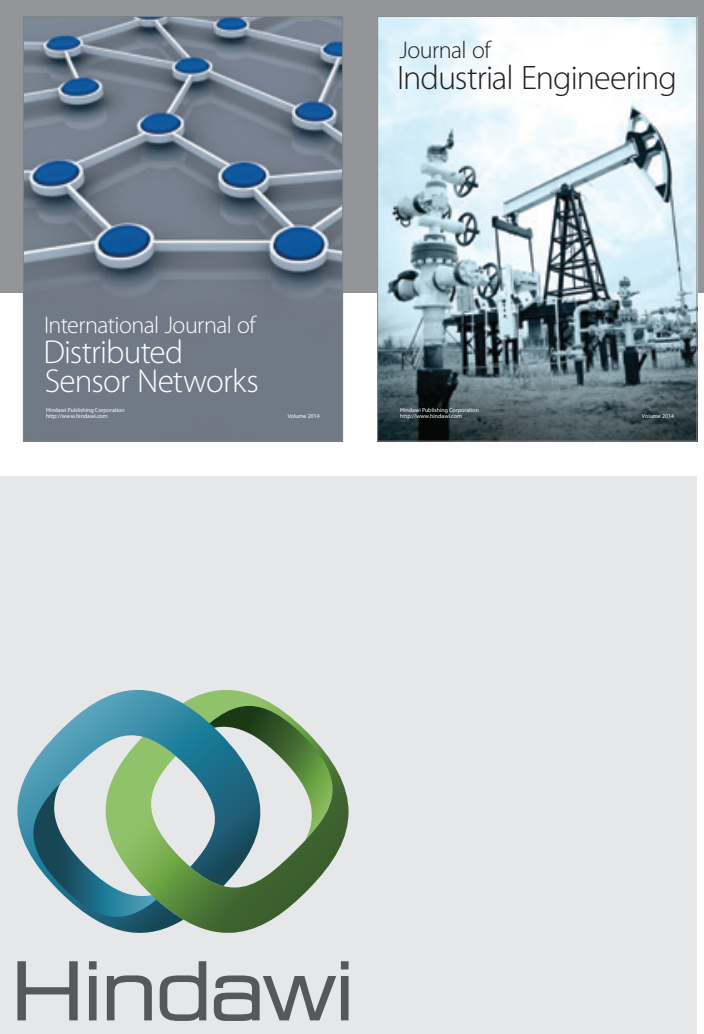

Submit your manuscripts at

http://www.hindawi.com

\section{Computer Networks} and Communications
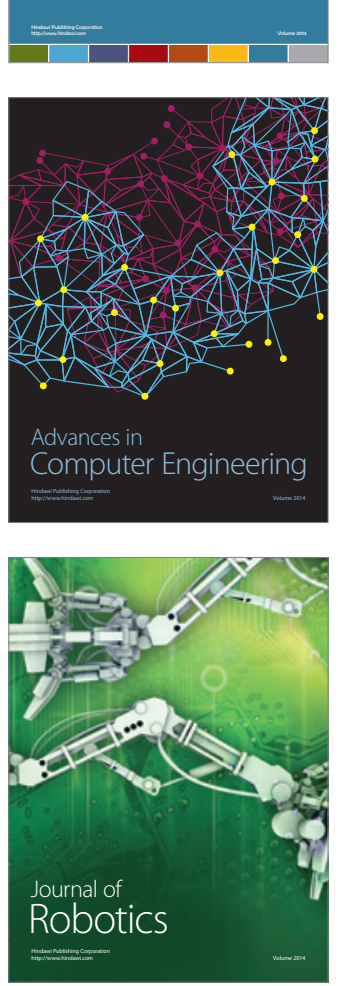
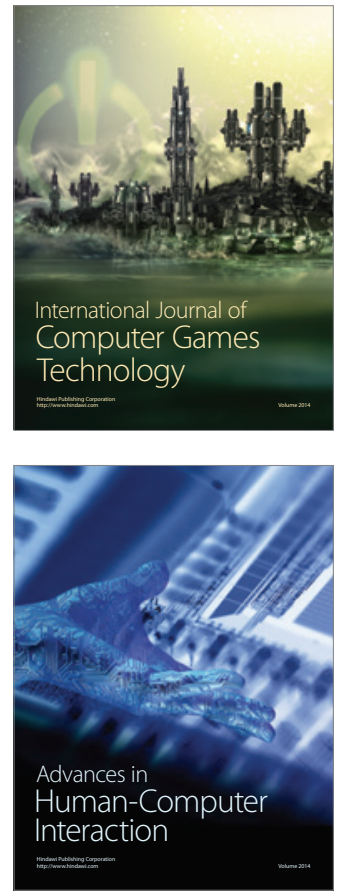
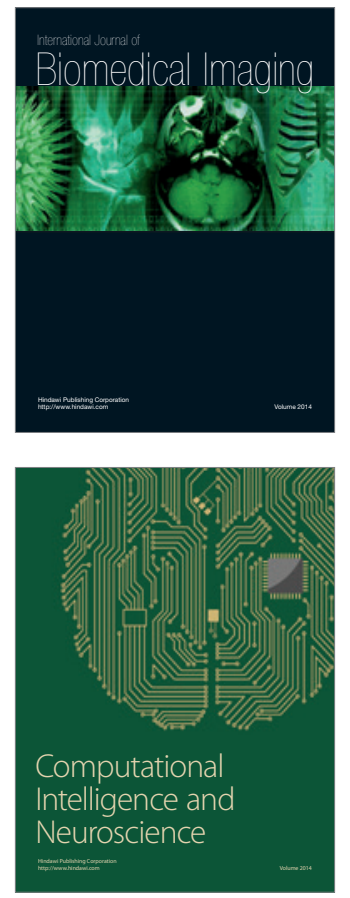
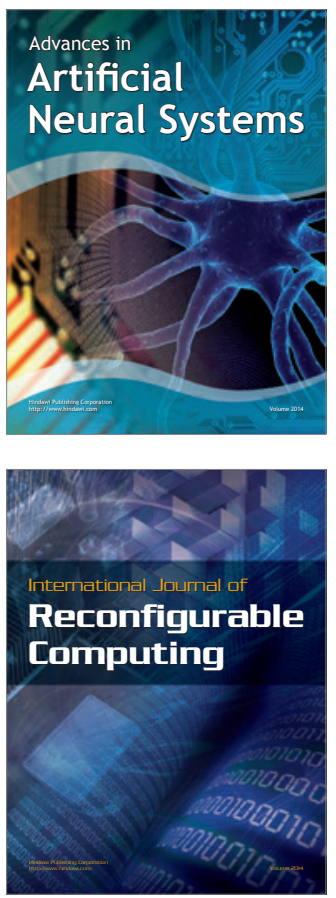
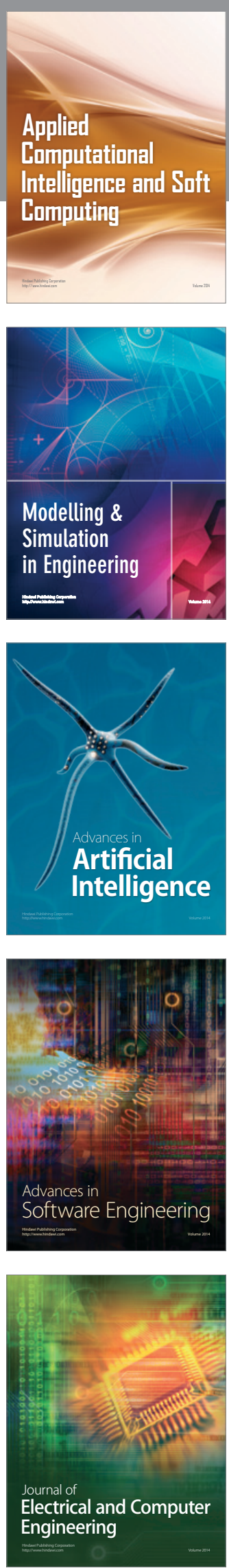